Pacific Journal of Mathematics

POWER INVARIANT RINGS 


\section{POWER INVARIANT RINGS}

\section{JoONG-Ho KIM}

$A$ ring $A$ is called power invariant if whenever $B$ is a ring such that the formal power series rings $A[[X]]$ and $B[[X]]$ are isomorphic, then $A$ and $B$ are isomorphic. $A$ ring $A$ is said to be strongly power invariant if whenever $B$ is a ring and $\phi$ is an isomorphism of $A[[X]]$ onto $B[[X]]$, then there exists a $B$-automorphism $\psi$ of $B[[X]]$ such that $\psi(X)=\phi(X)$. Strongly power invariant rings are power invariant. For any commutative ring $A, A / J(A)^{n}$ is strongly power invariant, where $J(A)$ is the Jacobson radical of $A$, and $n$ is any positive integer. A left or right Artinian ring is strongly power invariant. If $A$ is a left or right Noetherian ring, then $A[t]$, the polynomial ring in an indeterminate $t$ over $A$, is strongly power invariant.

Introduction. Coleman and Enochs [2] raised the following question: Can there be nonisomorphic rings $A$ and $B$ whose polynomial rings $A[X]$ and $B[X]$ are isomorphic? Recently Hochster [4] answered this question in the affirmative. The analogous question about a commutative formal power series ring was raised by O'Malley [7]: If $A[[X]] \cong B[[X]]$, must $A \cong B$ ? We know no counterexamples.

In this paper all rings are assumed to have identity elements. The Jacobson radical and the prime radical (the intersection of all prime ideals) of a ring $A$ will be denoted by $J(A)$ and $\operatorname{rad}(A)$, respectively. Let $A[[X]]$ be the formal power series ring in a commutative indeterminate $X$ over a ring $A$, and let $\beta$ be a central element of $A[[X]]$. Then $\left(\beta^{n}\right)$ will denote the ideal of $A[[X]]$ generated by $\beta^{n}$ for a nonnegative integer $n$, and $(A[[X]],(\beta))$ denotes the topological ring $A[[X]]$ with the $(\beta)$-adic topology. It is well known that $(A[[X]],(\beta))$ is Hausdorff if and only if $\bigcap_{n=1}^{\infty}\left(\beta^{n}\right)=(0)$. The $(\beta)$-adic topology is metrizable in the obvious way, and we say that $(A[[X]],(\beta))$ is complete if each Cauchy sequence of $A[[X]]$ converges in $A[[X]]$. Then clearly $(A[[X]],(X))$ is a complete Hausdorff space. Extending the terminology used in [2], O'Malley [7] defined "power invariant ring" and "strongly power invariant ring" as follows: A ring $A$ is power invariant if whenever $B$ is a ring such that $A[[X]] \cong B[[X]]$, then $A \cong B$. A ring $A$ is said to be strongly power invariant if whenever $B$ is a ring and $\phi$ is an isomorphism of $A[[X]]$ onto $B[[X]]$, then there exists a $B$-automorphism $\psi$ of $B[[X]]$ such that $\psi(X)=\phi(X)$.

Let $A$ be a strongly power invariant ring and let $\phi$ be an isomorphism of $A[[X]]$ onto $B[[X]]$. Then there exists a $B$-automorphism 
$\psi$ of $B[[X]]$ such that $\psi(X)=\phi(X)$. Then $\psi^{-1} \phi$ is an isomorphism of $A[[X]]$ onto $B[[X]]$ such that $\left(\psi^{-1} \phi\right)(X)=X$. Therefore, $A \cong$ $A[[X]] /(X) \cong B[[X]] /(X) \cong B$. Thus a strongly power invariant ring is power invariant.

In this paper we attempt to impose conditions on a ring $A$ so that $A[X] \cong B[[X]]$ implies $A \cong B$.

1. Strongly power invariant rings. The following theorem extends Theorem (4.5) in [8].

TheOREM 1.1. Let $B$ be a ring and $\beta=\sum_{i=0}^{\infty} b_{i} X^{i}$, an element of $B[[X]]$. Then the following statements are equivalent:

(1) $b_{i}$ is central for each $i, b_{1}$ is a unit, and $(B[[X]],(\beta))$ is a complete Hausdorff space.

(2) There exists a B-automorphism of $\psi$ of $B[[X]]$ such that $\psi(X)=\beta$.

Proof. Suppose that (2) holds. Since $(B[[X]],(X))$ is a complete Hausdorff space and $\psi$ is a uniformly bicontinuous mapping of $(B[[X]],(X))$ onto $(B[[X]],(\beta)),(B[[X]],(\beta))$ is a complete Hausdorff space. Since $X$ commutes with every element of $B, \beta$ commutes with any element of $B$ and therefore $b_{i}$ is central for each $i$. Let $C$ be the center of $B$. Then $C[[X]]$ is the center of $B[[X]]$ and hence $\phi(C[[X]])=C[[X]]$. Then $\psi$ induces the $C$-automorphism of $C[[X]]$ which maps $X$ onto $\beta$. Therefore, by Theorem (4.5) in [8], $b_{1}$ is a unit. Thus (2) implies (1).

Suppose that $(1)$ holds. Since $(B[[X]],(\beta))$ is a complete Hausdorff space, there is a $B$-endomorphism $\psi$ of $B[[X]]$ such that $\psi(X)=\beta$. This comes from the same argument as the commutative case; namely (2.2) in [8]. Since $b_{i}$ is central for each $i$, that $\psi$ is a $B$-automorphism, also follows from the commutative argument; namely Lemma (4.2) and Corollary (4.4) in [8]. This completes the proof.

Let $\phi$ be an isomorphism of $A[[X]]$ onto $B[[X]]$ such that $\phi(X)=$ $\beta=\sum_{i=0}^{\infty} b_{i} X^{i}$. By similar argument as in the proof of Theorem 1.1, we see that $b_{i}$ is central in $B$ for each $i$ and $(B[[X]],(\beta))$ is a complete Hausdorff space. Therefore, by Theorem 1.1, we see that a ring $A$ is strongly power invariant if and only if whenever $B$ is a ring and $\phi$ is an isomorphism of $A[[X]]$ onto $B[[X]]$ such that $\phi(X)=$ $\sum_{i=0}^{\infty} b_{i} X^{i}$, then $b_{1}$ is a unit.

The following lemma has appeared as Result 4.3 in [7] for the commutative case.

Lemma 1.2. For any ring $A, A / J(A)$ is strongly power invariant. In particular, if $A$ is a semisimple ring then $A$ is strongly power invariant. 
Proof. Let $A$ be a semisimple. To prove this lemma, it suffices to show that $A$ is strongly power invariant. Let $B$ be a ring such that there is an isomorphism $\phi$ of $A[[X]]$ onto $B[[X]]$. Let $\phi(X)=$ $\sum_{i=0}^{\infty} b_{i} X^{i}$. Since $J(A)=(0)$, it follows that $J(A[[X]])=(X)$, and

$$
\phi(J(A[[X]]))=\phi((X))=(\phi(X))=\dot{\phi}(X) \cdot B[[X]]=J(B[[X]]) .
$$

Clearly $X \in J(B[[X]])$, and so there exists $\sum_{i=6}^{\infty} c_{i} X^{i} \in B[[X]]$ such that $\phi(X) \cdot \sum_{i=0}^{\infty} c_{i} X^{i}=X$; i.e., $\quad\left(\sum_{i=0}^{\infty} b_{i} X^{i}\right) \cdot\left(\sum_{i=0}^{\infty} c_{i} X^{i}\right)=X$. Then $b_{0} c_{1}+$ $b_{1} c_{0}=1$. But $b_{0} \in J(B)$, so $1-b_{0} c_{1}$ is a unit. Therefore, $b_{1} c_{0}$ is a unit, and so $b_{1}$ is a unit. Hence $A$ is strongly power invariant.

THEOREM 1.3. If $A$ is a commutative ring, then for any positive integer $n, A / J(A)^{n}$ is strongly power invariant.

Proof. Let $A$ be a commutative ring such that $J(A)$ is nilpotent. To prove this theorem, it suffices to show that $A$ is strongly power invariant. Let $B$ be a ring such that there is an isomorphism $\phi$ of $A[[X]]$ onto $B[[X]]$, and let $\phi(X)=\beta=\sum_{i=0}^{\infty} b_{i} X^{i}$. Then clearly $B$ is commutative. Let $N$ be the ideal of nilpotent elements of $B$, and let $\left\{P_{r}\right\}$ be the collection of prime ideals of $B$. Then $N=\bigcap_{r} P_{r}$, and for each $\gamma, P_{r}[[X]]$ is a prime ideal of $B[[X]]$. Therefore, the ideal of nilpotent elements of $B[[X]]$ is a subset of $N[[X]]$. Note that $N[[X]]$ is not necessarily the ideal of nilpotent elements of $B[[X]]$. Since $J(A)$ is nilpotent, $J(A)[[X]]$ is the ideal of nilpotent elements of $A[[X]]$. Therefore, $\phi(J(A)[[X]]) \subseteq N[[X]]$. In order to show the opposite inclusion, let $g=\sum_{i=0}^{\infty} g_{i} X^{i} \in N[[X]] ; g_{i} \in N$ for each $i$, and let $\phi^{-1}(X)=\alpha=\sum_{i=0}^{\infty} a_{i} X^{i}, a_{i} \in A$. Then $\phi^{-1}(g)=\sum_{i=0}^{\infty} \phi^{-1}\left(g_{i}\right) \alpha^{i}$, and $\phi^{-1}\left(g_{i}\right)$ is a nilpotent element of $A[[X]]$ for each $i$. Note that $a_{0} \in J(A)$ i.e., $a_{0}$ is nilpotent, and $\phi^{-1}\left(g_{i}\right) \in J(A)[[X]]$. Expanding $\sum_{i=0}^{\infty} \phi^{-1}\left(g_{i}\right) \alpha^{i}$ in powers of $X$, we see that the coefficient of $X^{i}$ is an element of $J(A)$ for each $i$ since $a_{0}$ is nilpotent. Thus $\phi^{-1}(g) \in J(A)[[X]]$. Therefore, we get $\phi(J(A))[[X]]=N[[X]]$. Consider the isomorphism $\bar{\phi}$ : $(A / J(A))[[X]] \rightarrow(B / N)[[X]]$ given by

$(A / J(A))[[X]] \longrightarrow A[[X]] / J(A)[[X]] \longrightarrow B[[X]] / N[[X]] \longrightarrow(B / N)[[X]]$

where the middle isomorphism is induced by $\phi$ and others are the obvious ones. Then it follows that $\bar{\phi}(X)=\sum_{i=0}^{\infty} \bar{b} X^{i}$, where $\bar{b}_{\imath}$ denotes the coset $b_{\imath}+N$ in $B / N$. Since $A / J(A)$ is strongly power invariant, $\bar{b}_{1}$ is a unit in $B / N$. But $N \cong J(B)$ so $b_{1}$ is a unit in $B$. Thus $A$ is strongly power invariant. This completes the proof.

Corollary 1.4. Let $A$ be a ring and $C$, the center of $A$. If $J(C)$ is nilpotent, then $A$ is strongly power invariant. In particular, if $C$ is a Artinian ring, then $A$ is strongly power invariant. 
Proof. Let $B$ be a ring such that there is an isomorphism $\phi$ of $A[[X]]$ onto $B[[X]]$, and let $\phi(X)=\sum_{i=0}^{\infty} b_{i} X^{i}$. If $D$ denotes the center of $B, \phi(C[[X]])=D[[X]]$. But by Theorem 1.3, $C$ is strongly power invariant. Therefore, $b_{1}$ is a unit and so $A$ is strongly power invariant.

It is well known that the prime radical of a ring $A$, denoted by $\operatorname{rad}(A)$, is the intersection of all prime ideals of $A$, and also it is the ideal of all strongly nilpotent elements of $A$. (P. 55-56 in [6].) Clearly, every strongly nilpotent element is nilpotent. In particular, if $A$ is commutative, then every nilpotent element is strongly nilpotent. Note that if $A$ is a commutative Noetherian ring, and $N$ is the ideal of nilpotent elements of $A$, then $N[[X]]$ is the ideal of nilpotent elements of $A[[X]]$ [3]. The following lemma extends this statement to the noncommutative case.

Lemma 1.5. If $A$ is a left or right Noetherian ring, then $\operatorname{rad}(A[[X]])=\operatorname{rad}(A)[[X]]$.

Proof. We show that if $P$ is a prime ideal of $A$, then $P[[X]]$ is a prime ideal of $A[[X]]$. Suppose that $P$ is a prime ideal of $A$ and $P[[X]]$ is not a prime ideal of $A[[X]]$. Then there exist $f=\sum_{i=0}^{\infty} f_{i} X^{i}$ and $g=\sum_{i=0}^{\infty} g_{i} X^{i}$ in $A[[X]]$ such that $f \cdot A[[X]] \cdot g \subseteq P[[X]]$ but $f \notin P[[X]]$ and $g \notin P[[X]]$. Let $m$ be the smallest integer such that $f_{m} \notin P$, and let $n$ be the smallest integer such that $g_{n} \notin P$. Since $f \cdot A[[X]] \cdot g \subseteq P[[X]], f \cdot a \cdot g$ belongs to $P[[X]]$ for any element $a$ of $A$. Expanding $f \cdot a \cdot g$ in powers of $X$, we see that the coefficient of $X^{m+n}$ is $\sum_{i+j=0}^{m+n} f_{i} a g_{j}$ which is in $P$. But $\sum_{i+j=0}^{m+n} f_{i} a g_{j}-f_{m} a g_{n} \in P$, so $f_{m} a g_{n}$ must be in $P$. Therefore, $f_{m} A g_{n} \leqq P$, but $P$ is a prime ideal of $A$; so $f_{m} \in P$ or $g_{n} \in P$. This is a contradiction to our choice of $m$ and $n$. Hence $P[[X]]$ is a prime ideal of $A[[X]]$. Therefore, it follows that $\operatorname{rad}(A[[X]]) \subseteq \operatorname{rad}(A)[[X]]$. To show the opposite inclusion, we let $\sum_{i=0}^{\infty} a_{i} X^{i} \in \operatorname{rad}(A)[[X]]$. Then each $a_{i}$ is strongly nilpotent. Let $\mathfrak{A}$ be the ideal of $A$ generated by the set of all $a_{i}$ 's. Then clearly $\mathfrak{A} \subseteq \operatorname{rad}(A)$; therefore, $\mathfrak{A}$ is a nil ideal of $A$. But since $A$ is left or right Noetherian, $\mathfrak{Q}$ is nilpotent. Thus $\sum_{i=0}^{\infty} a_{i} X^{i} \in \operatorname{rad}(A[[X]])$. Therefore, $\operatorname{rad}(A[[X]])=\operatorname{rad}(A)[[X]]$.

THEOREM 1.6. Let $A$ be a left or right Noetherian ring and let $N=\operatorname{rad}(A)$. Then $A$ is strongly power invariant if $A / N$ is strongly power invariant.

Proof. Let $B$ be a ring such that there is an isomorphism $\phi$ of $A[[X]]$ onto $B[[X]]$, and let $M=\operatorname{rad}(B)$. Since $A$ is left (or right) Noetherian, $A[[X]]$ is left (or right) Noetherian. Then $B[[X]]$ is left 
(or right) Noetherian, and therefore, $B$ is left (or right) Noetherian. So $\operatorname{rad}(B[[X]])=M[[X]]$ (by Lemma 1.5). From the invariance of the prime radical under isomorphism, we have that $\phi(N[[X]])=$ $M[[X]]$. Write $\phi(X)=\sum_{i=0}^{\infty} b_{i} X^{i} ; \quad b_{\imath} \in B$. Consider the isomorphism, $\bar{\phi}:(A / N)[[X]] \rightarrow(B / M)[[X]]$ given by

$$
(A / N)[[X]] \longrightarrow A[[X]] / N[[X]] \longrightarrow B[[X]] / M[[X]] \longrightarrow(B / M)[[X]],
$$

where the middle isomorphism is induced by $\phi$ and the others are the obvious ones. Since $A / N$ is strongly power invariant, we can show that $b_{1}$ is a unit of $B$ by the same argument as in the proof of Theorem 1.3. Thus $A$ is a strongly power invariant ring.

COROLlary 1.7. If $A$ is a left or right Noetherian ring such that $J(A)$ is nil, then $A$ is strongly power invariant.

Proof. Clearly $J(A)$ is nilpotent. So every element of $J(A)$ is strongly nilpotent. Therefore, $J(A)=\operatorname{rad}(A)$. By Lemma 1.2 and Theorem 1.6, $A$ is strongly power invariant.

COROLlary 1.8. A left or right Artinian ring is strongly power invariant.

COROLlary 1.9. If $A$ is a left or right Noetherian ring and if $A[t]$ is the polynomial ring in a commutative indeterminate $t$ over $A$, then $A[t]$ is strongly power invariant.

Proof. It is well known that for any ring $A, J(A[t])=N[t]$ holds, where $N=J(A[t]) \cap A$ and $N$ is a nil ideal in $A$ [1]. Since $A$ is left (or right) Noetherian, $N$ is nilpotent and $A[t]$ is left (or right) Noetherian. Thus $J(A[t])=N[t]$ is a nilpotent ideal in $A[t]$. Therefore, by Corollary $1.7, A[t]$ is strongly power invariant.

2. Perfect power invariant rings. The following proposition extends Theorem 3.1 in [7].

Proposition 2.1. Let $A$ and $B$ be rings and suppose that $\phi$ is an isomorphism of $A[[X]]$ onto $B[[X]]$. If $\phi(A) \subseteq B$, then $\phi(A)=B$.

Proof. Let $\phi(X)=\beta=\sum_{i=0}^{\infty} b_{i} X^{i} ; b_{i} \in B$. Then $b_{i}$ is central for each $i$ and $(B[[X]],(\beta))$ is a complete Hausdorff space. Then there exists a $B$-endomorphism $\psi$ of $B[[X]]$ into $B[[X]]$ such that $\psi(X)=$ $\beta$. Then by hypothesis, we have

$$
B[[X]]=\phi(A)[[\beta]] \subseteq B[[\beta]] \subseteq B[[X]] .
$$


Therefore, $B[[\beta]]=B[[X]]$, which implies $\psi$ is onto. Now let $\bar{B}$ be $B /\left(b_{1}\right)$ and let $\bar{b}=b+\left(b_{1}\right)$ for $b \in B$. Then $X \rightarrow \sum_{i=0}^{\infty} \bar{b}_{i} X^{i}$ induces a surjective $\bar{B}$-endomorphism of $\bar{B}[[X]]$. But $\bar{b}_{1}$ is 0 , so this impossible unless $\left(b_{1}\right)=B$; i.e., $b_{1}$ is a unit. Therefore, by Theorem $1.1, \psi$ is a $B$-automorphism of $B[[X]]$. Then $\psi^{-1} \phi$ is an isomorphism of $A[[X]]$ onto $B[[X]]$ such that $\psi^{-1} \phi(A) \subseteq B$ and $\psi^{-1} \phi(X)=X$. So $\psi^{-1} \phi(A)=$ $B$; but $\psi^{-1}(B)=B$; therefore $\phi(A)=B$.

Definition. A ring $A$ is said to be perfectly power invariant if whenever $B$ is a ring and $\phi$ is an isomorphism of $A[[X]]$ onto $B[[X]]$, then $\phi(A) \subseteq B$.

Let $A$ be a perfectly power invariant ring, and let $B$ be a ring such that there is an isomorphism $\phi$ of $A[[X]]$ onto $B[[X]]$. In the proof of Proposition 2.1, we have shown that there exists a $B$-automorphism $\psi$ of $B[[X]]$ such that $\psi(X)=\phi(X)$. So a perfectly power invariant ring is strongly power invariant. But a strongly power invariant ring is not necessarily perfectly power invariant.

EXAMPLE. Let $K$ be a field and let $K[t]$ be the polynomial ring in an indeterminate $t$ over $K$ then $K[t]$ is strongly power invariant (by Corollary 1.9). But, by Corollary 2.8 in [5], we see that there is an automorphism $\phi$ of $K[t][[X]]$ such that $\phi(K[t]) \nsubseteq K[t]$. Therefore, $K[t]$ is not perfectly power invariant.

Proposition 2.2. If a ring $A$ is generated by its central idempotents, then $A$ is perfectly power invariant. In particular a Boolean ring is perfectly power invariant.

Proof. Let $B$ be a ring such that there is an isomorphism $\phi$ of $A[[X]]$ onto $B[[X]]$. It is straightforward to show that the only central idempotents of $B[[X]]$ are those of $B$, therefore $\phi(A) \cong B$. Thus $B$ is perfectly power invariant.

Proposition 2.3. Let $K$ be a field and let $\Pi$ be the prime field of $K$. If $K$ is algebraic over $\Pi$, then $K$ is perfectly power invariant.

Proof. Let $B$ be a ring such that there is an isomorphism $\phi$ of $K[[X]]$ onto $B[[X]]$. Since $K$ is strongly power invariant, we have $K \cong B$. Therefore, $B$ is a field. Clearly, $\phi(\Pi)$ is the prime field of $B$. It is straightforward to show that any element $f \in B[[X]] ; f \notin B$, is not algebraic over a field $B$. So $f$ is not algebraic over $\phi(I I)$. But $\phi(K)$ is algebraic over $\phi(\Pi)$, therefore $\phi(K) \subseteq B$. Thus $K$ is perfectly power invariant. 
Corollary 2.4. Let $D$ be an integral domain and let $I$ be the prime ring of $D$ (that is, II is the subring of $D$ generated by the identity element of $D$ ). If $D$ is integral over $\Pi$, then $D$ is perfectly power invariant.

COROLLARY 2.5. An algebraic number field is perfectly power invariant, and the ring of algebraic integers is perfectly power invariant.

\section{REFERENCES}

1. S. A. Amitsur, Radicals of polynomial rings, Canad. J. Math., 8 (1956), 355-361.

2. D. B. Coleman and E. E. Enochs, Isomorphic polynomial rings, Proc. Amer. Math. Soc., 27 (1971), 247-252.

3. D. E. Fields, Zero divisors and nilpotent elements in power series rings, Proc. Amer. Math. Soc., 27 (1971), 427-433.

4. M. Hochster, Nonuniqueness of coefficient rings in a polynomial ring, Proc. Amer. Math. Soc., 34 (1972), 81-82.

5. Joong-Ho Kim, R-automorphism of $R[t][[X]]$, Pacific J. Math., 42 (1972), 81-88.

6. J. Lambek, Lectures on Rings and Modules, Blaisdell, 1966.

7. M. J. O'Malley, Isomorphic power series rings, Pacific J. Math., 41 (1972), 503-512.

8. M. J. O'Malley and C. Wood, R-endomorphisms of $R[[X]]$, J. Algebra, 15 (1970), 314-327.

Received November 15, 1972. This research was supported by the East Carolina University Research Council.

EAST CaRoLina UNIVERSity 



\section{PACIFIC JOURNAL OF MATHEMATICS}

\section{EDITORS}

RICHARD ARENS (Managing Editor)

University of California

Los Angeles, California 90024

R. A. BeAumont

University of Washington

Seattle, Washington 98105
J. DugundJI*

Department of Mathematics

University of Southern California

Los Angeles, California 90007

D. Gilbarg and J. Milgram

Stanford University

Stanford, California 94305

\section{ASSOCIATE EDITORS}

E. F. BECKENBACH

B. H. NEUMANN

F. WOLF

K. YoSHIDA

\section{SUPPORTING INSTITUTIONS}

UNIVERSITY OF BRITISH COLUMBIA
CALIFORNIA INSTITUTE OF TECHNOLOGY
UNIVERSITY OF CALIFORNIA
MONTANA STATE UNIVERSITY
UNIVERSITY OF NEVADA
NEW MEXICO STATE UNIVERSITY
OREGON STATE UNIVERSITY
UNIVERSITY OF OREGON
OSAKA UNIVERSITY

UNIVERSITY OF BRITISH COLUMBIA CALIFORNIA INSTITUTE OF TECHNOLOGY UNIVERSITY OF CALIFORNIA MONTANA STATE UNIVERSITY NEW MEXICO STATE UNIVERSITY UNIVERSITY OF OREGON OSAKA UNIVERSITY
UNIVERSITY OF SOUTHERN CALIFORNIA STANFORD UNIVERSITY UNIVERSITY OF TOKYO UNIVERSITY OF UTAH WASHINGTON STATE UNIVERSITY UNIVERSITY OF WASHINGTON AMERICAN MATHEMATICAL SOCIETY NAVAL WEAPONS CENTER

* C. R. DePrima California Institute of Technology, Pasadena, CA 91109, will replace J. Dugundji until August 1974. 


\section{Pacific Journal of Mathematics}

\section{Vol. 51, No. $1 \quad$ November, 1974}

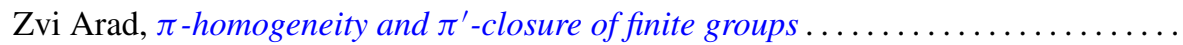

Ivan Baggs, A connected Hausdorff space which is not contained in a maximal

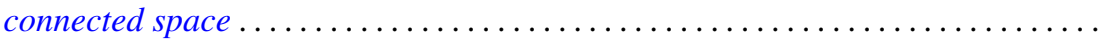

Eric Bedford, The Dirichlet problem for some overdetermined systems on the unit ball in $C^{n}$

R. H. Bing, Woodrow Wilson Bledsoe and R. Daniel Mauldin, Sets generated by

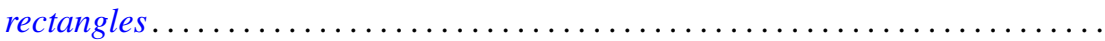

Carlo Cecchini and Alessandro Figà-Talamanca, Projections of uniqueness for

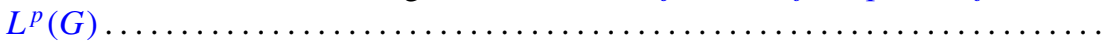

Gokulananda Das and Ram N. Mohapatra, The non absolute Nörlund summability of Fourier series .

Frank Rimi DeMeyer, On separable polynomials over a commutative ring ........ Richard Detmer, Sets which are tame in arcs in $E^{3} \ldots \ldots \ldots \ldots \ldots \ldots \ldots \ldots$

William Erb Dietrich, Ideals in convolution algebras on Abelian groups ..........

Bryce L. Elkins, A Galois theory for linear topological rings .................

William Alan Feldman, A characterization of the topology of compact convergence on $C(X)$.

Hillel Halkin Gershenson, A problem in compact Lie groups and framed cobordism

Samuel R. Gordon, Associators in simple algebras.

Marvin J. Greenberg, Strictly local solutions of Diophantine equations

Jon Craig Helton, Product integrals and inverses in normed rings . . . . . . . . . . . .

Domingo Antonio Herrero, Inner functions under uniform topology . . .

Jerry Alan Johnson, Lipschitz spaces .

Marvin Stanford Keener, Oscillatory solutions and multi-point boundary value

functions for certain nth-order linear ordinary differential equations.

John Cronan Kieffer, A simple proof of the Moy-Perez generalization of the

Shannon-McMillan theorem .......................

Joong Ho Kim, Power invariant rings

Gangaram S. Ladde and V. Lakshmikantham, On flow-invariant sets .

Roger T. Lewis, Oscillation and nonoscillation criteria for some self-adjoint even

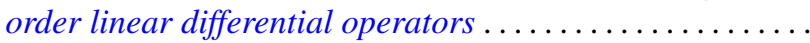

Jürg Thomas Marti, On the existence of support points of solid convex sets ..

John Rowlay Martin, Determining knot types from diagrams of knots . .

James Jerome Metzger, Local ideals in a topological algebra of entire functions

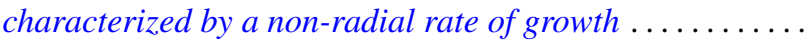

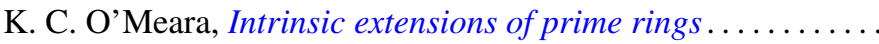

Stanley Poreda, A note on the continuity of best polynomial approximations ..

Robert John Sacker, Asymptotic approach to periodic orbits and local prolongations

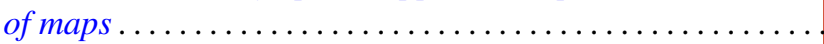

Eric Peter Smith, The Garabedian function of an arbitrary compact set . .

Arne Stray, Pointwise bounded approximation by functions satisfying a side condition

John St. Clair Werth, Jr., Maximal pure subgroups of torsion complete abelian

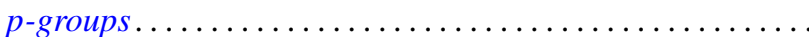

